\title{
Satisfação com Aparelhos de Amplificação Sonora Individual entre usuários de serviços de saúde auditiva
}

\section{Satisfaction with hearing aids among users of hearing health}

\section{services}

\author{
Mirna Rossi Barbosa', Daniel de Sousa Medeiros², Gisele Marques Ribeiro33, Luiza Augusta Rosa Rossi-Barbosa4, \\ Antônio Prates Caldeira ${ }^{4}$
}

\begin{abstract}
RESUMO
Objetivo: Conhecer a satisfação com Aparelho de Amplificação Sonora Individual entre usuários assistidos pelo serviço de saúde auditiva do Norte de Minas, identificando os fatores associados e a percepção sobre a assistência. Métodos: Trata-se de um estudo transversal, exploratório, conduzido no Norte de Minas Gerais, com 302 indivíduos assistidos pelo Serviço de Atenção à Saúde Auditiva, que responderam a um questionário de avaliação do serviço de saúde auditiva e satisfação com o aparelho. Foi aplicada regressão logística para verificar a associação entre as variáveis sóciodemográficas e a satisfação com o aparelho. Resultados: A média de satisfação com o aparelho foi 5,09 e de avaliação do serviço foi 7,25, em uma escala de zero a dez. Na época da entrevista, $46 \%$ dos entrevistados relataram que não estavam fazendo uso do aparelho há mais de um mês, sendo que a maioria alegou dificuldades na adaptação. Os pacientes que tiveram dúvidas em relação ao uso do aparelho, apresentaram menores escores de satisfação. Conclusão: Os pacientes assistidos pelo Serviço Único de Saúde, de uma forma geral, alcançaram um indicador de satisfação regular com o aparelho e um indicador bom com o serviço ofertado.
\end{abstract}

Descritores: Avaliação de serviços de saúde; Indicadores de Serviços; Sistema Único de Saúde; Satisfação do Paciente; Perda Auditiva; Auxiliares de Audição

\begin{abstract}
Purpose: To know the satisfaction with individual hearing aids among users assisted by the hearing care service of northern Minas, identifying the related factors and the perception regarding the care. Methods: This was a cross-sectional, exploratory study conducted in Northern Minas Gerais, with 302 individuals assisted by the Service of Hearing Health Care, who responded to a questionnaire assessing the hearing health service and satisfaction with the device. Logistic regression was used to assess the association between the sociodemographic variables and the satisfaction with the device. Results: The mean satisfaction with the device was 5.09 and of the service evaluation was 7.25 , on a scale of zero through ten. At the time of the interview, $46 \%$ of interviewees reported that they were not using the device for over a month, and the majority claimed difficulties in adaptation. Patients who had questions about the use of the device exhibited lower satisfaction scores. Conclusion: Patients assisted by the Unified Health System, in general, reached an indicator of regular satisfaction with the device and a good indicator with the service offered.
\end{abstract}

Keywords: Health Services Evaluation; Indicators of Health Services; Unified Health System; Patient Satisfaction; Hearing Loss; Hearing Aids

Trabalho realizado no Programa de Pós-Graduação em Ciências da Saúde, Universidade Estadual de Montes Claros - UNIMONTES - Montes Claros (MG), Brasil, com bolsas concedidas pela Coordenação de Aperfeiçoamento de Pessoal de Nível Superior (CAPES) e Fundação de Amparo à Pesquisa do Estado de Minas Gerais (FAPEMIG).

(1) Programa de Pós-graduação (Mestrado) em Ciências da Saúde, Universidade Estadual de Montes Claros - UNIMONTES - Montes Claros (MG), Brasil.

(2) Superintendência Regional de Ensino de Montes Claros, Secretaria de Educação de Minas Gerais - Montes Claros (MG), Brasil.

(3) Programa de Pós-graduação (Especialização) em Saúde da Família, Departamento de Enfermagem, Universidade Estadual de Montes Claros - UNIMONTES - Montes Claros (MG), Brasil.

(4) Programa de Pós-graduação (Doutorado) em Ciências da Saúde, Universidade Estadual de Montes Claros - UNIMONTES - Montes Claros (MG), Brasil. Conflito de interesses: não

Contribuição dos autores: $M R B$ pesquisadora principal, elaboração da pesquisa, elaboração do cronograma, levantamento da literatura, coleta e análise dos dados, redação do artigo, submissão e trâmites do artigo; $D S M$ coautor, levantamento da literatura, coleta dos dados, redação do artigo; GMR coautora, levantamento da literatura, coleta dos dados, redação do artigo; $L A R R B$ coautora, levantamento da literatura, coleta e análise dos dados, redação do artigo; $A P C$ orientador, elaboração da pesquisa, análise dos dados, correção da redação do artigo, aprovação da versão final.

Endereço para correspondência: Mirna Rossi Barbosa. Gabinete 4, Av. Cula Mangabeira, 562, Santo Expedito, Montes Claros (MG), Brasil, CEP: $39401-001$. E-mail: mirnarossi@ hotmail.com

Recebido em: 3/5/2013; Aceito em: 11/9/2013 


\section{INTRODUÇÃO}

De acordo com o Instituto Brasileiro de Geografia e Estatística (IBGE) $)^{(1)}$, 9,7 milhões de brasileiros possuem algum tipo de perda auditiva. Com o aumento da expectativa de vida, estima-se que esse número possa ser ainda maior, já que a função auditiva tende a se deteriorar com a idade ${ }^{(2)}$. A maior dificuldade decorrente da perda auditiva refere-se às habilidades de reconhecimento de fala ${ }^{(3)}$, o que prejudica a comunicação do indivíduo e suas relações sociais ${ }^{(4,5)}$.

Nos últimos anos, no Brasil, o Sistema Único de Saúde (SUS) ampliou o acesso aos cuidados audiológicos, com a implantação de redes de atenção ao paciente com perda auditiva ${ }^{(6)}$, que contemplam desde a promoção da saúde auditiva até a terapia fonoaudiológica. Há necessidade de pesquisas que avaliem o impacto de tais serviços e os efeitos proporcionados pelo uso do Aparelho de Amplificação Sonora Individual $(\mathrm{AASI})^{(7)}$, como forma de garantir a eficácia do tratamento ${ }^{(8)}$.

Os questionários de autoavaliação têm sido utilizados em diversos serviços, auxiliando no controle do desempenho dos usuários, durante o processo de seleção e adaptação do AASI ${ }^{(9)}$. Em 2000, foi proposto um questionário contendo sete itens de autoavaliação da satisfação com a amplificação sonora, visando alcançar dados comparáveis de diferentes investigações e modelos de serviços clínicos, denominado International Outcome Inventory for Hearing Aids (IOI-HA) ${ }^{(10)}$. Tal questionário foi traduzido para diferentes países. Todavia, esse questionário, no Brasil, foi considerado de difícil compreensão para pessoas com baixo nível sociocultural ${ }^{(11)}$, que talvez não consigam respondê-lo sem o auxílio de um profissional do serviço. Tal situação pode acarretar em respostas induzidas pelo pesquisador ${ }^{(12)}$. Além disso, é preciso destacar que os estudos têm utilizado questionários de autoavaliação para aferição da satisfação do usuário, mas esses questionários não são suficientes para avaliar a qualidade do serviço prestado ${ }^{(13)}$.

No contexto de construção de indicadores de saúde auditiva, um questionário de Avaliação do Serviço de Saúde Auditiva e Uso do Aparelho de Amplificação Sonora Individual (AASI) foi publicado recentemente, com perguntas que avaliam, além das atividades assistenciais, a satisfação do usuário ${ }^{(7)}$. No presente estudo, o objetivo foi conhecer a satisfação com AASI entre usuários assistidos pelo serviço de saúde auditiva, identificando os fatores associados e a percepção sobre a assistência.

\section{MÉTODOS}

Trata-se de um estudo transversal, exploratório, quantitativo, conduzido no Norte de Minas Gerais, região que abrange 86 municípios, com população estimada em 1,5 milhão de habitantes. Nesse cenário, realizou-se o levantamento da população assistida pelo Serviço de Atenção à Saúde Auditiva na Média e Alta Complexidade Ambulatorial, no período de janeiro de 2010 a agosto de 2011, período que representa o início do trabalho efetivo da Junta Reguladora de Saúde Auditiva (JRSA), com tempo mínimo de quatro meses de recebimento do AASI.

A partir da população assistida, efetuou-se a seleção de amostra aleatória simples de usuários maiores de 18 anos. O cálculo amostral foi realizado considerando o total de indivíduos assistidos, margem de erro de 5\%, nível de confiança de $95 \%$ e frequência do evento esperado de insatisfação de $30 \%$, com base em outros estudos ${ }^{(14)}$.

Foram excluídos pacientes com grave limitação da capacidade de compreensão e expressão para responder aos questionários e pacientes que não foram localizados em suas residências, após três tentativas.

A amostra contou com 302 pacientes atendidos pelo Serviço de Atenção à Saúde Auditiva na Média e Alta Complexidade Ambulatorial da região, provenientes de 33 municípios de oito microrregiões do Norte de Minas (Figura 1).

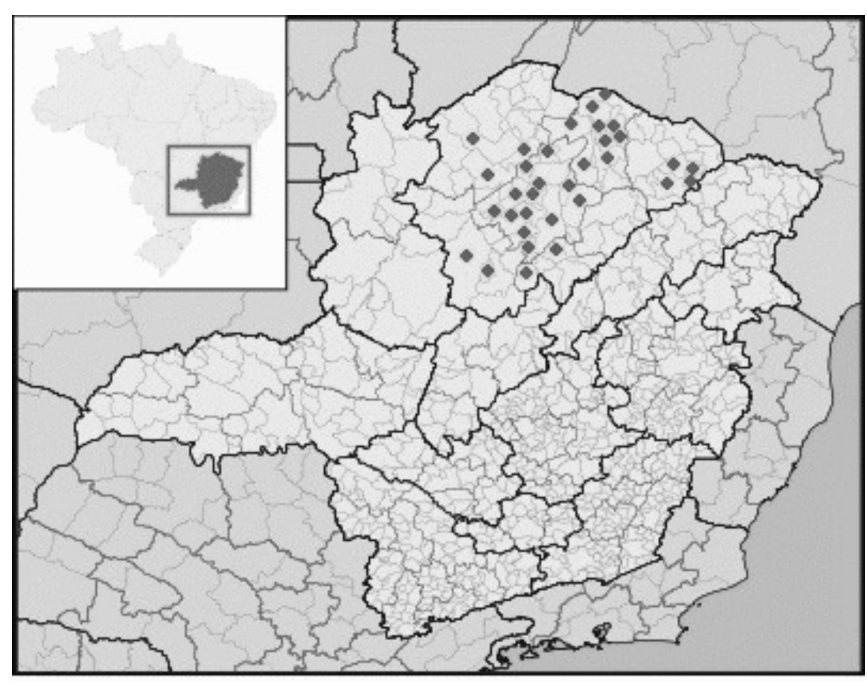

Adaptado de: Google Imagens

Figura 1. Mapa de Minas Gerais com municípios onde residiam os usuários entrevistados

A taxa de resposta foi de $98,7 \%$. Quanto ao gênero, 168 indivíduos $(55,6 \%)$ eram do gênero feminino e $134(44,4 \%)$ do gênero masculino. A maioria da população que procurou o serviço de saúde auditiva era constituída por pessoas idosas, sendo que $131(43,4 \%)$ tinham idade entre 60 e 79 anos, 83 (27,5\%), acima de 80 anos e $88(29,1 \%)$, entre 19 e 59 anos. Quanto à escolaridade, 73 (24,2\%) não eram alfabetizados, $143(47,4 \%)$ possuíam o ensino fundamental incompleto, 32 $(10,6 \%)$, o ensino fundamental completo, $38(12,6 \%)$, o ensino médio completo e 16 (5,3\%), o ensino superior completo. Com relação à ocupação, 200 indivíduos $(66,2 \%)$ eram aposentados, $36(11,9 \%)$ não trabalhavam ou estavam desempregados e 66 $(21,9 \%)$ trabalhavam. Não foi possível identificar o tipo e o grau da perda auditiva de todos os indivíduos, pois parte deles não tinha em mãos a cópia de seus exames audiométricos e a Secretaria Municipal de Saúde não dispunha dos dados de todos os usuários. Entre os pacientes que possuíam essas informações 
$(\mathrm{n}=160)$, o principal tipo de perda foi neurossensorial e o grau, moderado.

A coleta de dados foi realizada por meio de visitas domiciliares aos usuários residentes em zona urbana e zona rural, por pesquisadores que não possuíam vínculo com o serviço avaliado, com utilização do instrumento "Avaliação do Serviço de Saúde Auditiva e uso do AASI", questionário que avalia o serviço e a satisfação do usuário de AASI. O questionário é composto por 17 perguntas que avaliam globalmente o serviço, a acessibilidade e as necessidades do paciente. Dessas questões, quatro pontuam de 0 a 10 a avaliação do serviço e duas pontuam de 0 a 10 a satisfação do usuário. A avaliação do serviço refere ao atendimento de forma geral, a realização de testes com outros tipos e/ou marcas de aparelhos, o tempo de espera pelo aparelho e o(s) retorno(s) ao serviço, após o recebimento do aparelho. A satisfação do usuário é avaliada pelo tempo de uso do(s) aparelho(s) durante o dia e em quais momentos na vida do paciente o seu uso trouxe melhora. Os escores foram obtidos considerando-se as sugestões de mudanças em estudo prévio, realizado no norte de Minas Gerais $^{(7)}$, cujos pontos de corte em conceitos são: ruim (0 a 2), regular (3 a 5), bom (6 a 8), ótimo (9 e 10). O questionário possui, ainda, questões abertas que permitem a descrição dos motivos para o não uso do aparelho e satisfação ou insatisfação com o dispositivo.

As questões abertas relacionadas aos motivos para o não uso e à satisfação com o AASI, foram agrupadas em categorias e analisadas de forma descritiva. Na análise bivariada, os indicadores de satisfação com o AASI foram dicotomizados em satisfeitos (conceitos bom e ótimo) e insatisfeitos (conceitos ruim e regular). As variáveis sociodemográficas, as variáveis referentes ao AASI e outras três questões do questionário, também foram transformadas em variáveis dicotômicas. Foram incluídas no modelo múltiplo as variáveis que obtiveram valor de p menor que 0,30. Em seguida, foi realizada a análise de regressão logística, utilizando-se o programa SPSS ${ }^{\text {TM }}$ (Statistical Package for Social Sciences), versão 19.0, com nível de significância de 5\%. Também foi aplicado o teste de correlação de Spearman, para verificar a existência de correlação entre os indicadores de avaliação do serviço e de satisfação com o AASI.

Este estudo foi aprovado pelo Comitê de Ética em Pesquisa da Universidade Estadual de Montes Claros, sob o parecer 2888/11. Os indivíduos foram informados quanto aos objetivos, e concordaram com sua realização por meio da assinatura do Termo de Consentimento Livre e Esclarecido. Ao final da coleta de dados, nos casos em que houve necessidade, os entrevistados receberam orientações dos pesquisadores sobre diversos aspectos do uso e adaptação do AASI.

\section{RESULTADOS}

As características dos aparelhos indicados pelo Serviço de Atenção à Saúde Auditiva estão descritas na Tabela 1.
Tabela 1. Características dos Aparelhos de Amplificação Sonora Individual ofertados pelo SUS

\begin{tabular}{lcc}
\hline Variáveis & $\mathrm{n}$ & $\%$ \\
\hline Tipo de AASI & 236 & 78,1 \\
Retroauricular & 3 & 1,0 \\
Intrauricular & 58 & 19,2 \\
Intracanal & 5 & 1,7 \\
Microcanal & & \\
Categoria do AASI & & \\
C & 163 & 54,0 \\
B & 50 & 16,6 \\
A & 38 & 12,6 \\
Não informados & 51 & 16,9 \\
Adaptação & & \\
Bilateral & 273 & 90,7 \\
Unilateral & 28 & 9,3 \\
\hline
\end{tabular}

${ }^{*}$ Categoria C: digitais programáveis, não-lineares, compressão WDRC multicanal. Categoria B: digitais programáveis ou não, compressão WDRC mono ou multicanal. Categoria A: não programáveis, lineares, compressão monocanal

A grande maioria dos entrevistados $(81,1 \%)$ relatou que não fez testes com outras marcas de aparelho durante o processo de seleção do AASI e 53,6\% não retornaram ao Serviço para acompanhamento após um ano. As informações sobre o acesso e o atendimento aos usuários no Serviço de Atenção à Saúde Auditiva estão reproduzidas na Tabela 2.

Dos 302 indivíduos, 139 (46,0\%) não estavam fazendo uso do AASI, há mais de um mês, pelos seguintes motivos, conforme relato dos entrevistados: dificuldade para se adaptar com o aparelho, devido a desconforto, ruídos, dores na orelha, dor de cabeça e prurido $(n=57)$; defeitos técnicos no aparelho $(\mathrm{n}=25)$; ausência de benefício ( $\mathrm{n}=23)$; dificuldades financeiras, ou de acesso, para adquirir a pilha $(\mathrm{n}=16)$; dificuldades para manusear o aparelho $(n=7)$; defeitos no tubo ou no molde $(n=4)$; outros motivos $(n=7)$.

Dos 139 indivíduos que não estavam utilizando o AASI, 92 se disseram satisfeitos com o aparelho. Ao serem questionados sobre os motivos para estarem satisfeitos, 54,5\% alegaram que o aparelho os auxiliou na época em que faziam uso dele, 22,9\% disseram que o motivo foi o fato do aparelho ter sido gratuito e $22,6 \%$ relataram outros motivos.

As respostas dos entrevistados quanto ao uso e manutenção do AASI são apresentadas na Tabela 3.

A média de avaliação do serviço foi de 7,25 e a de satisfação com o AASI foi de 5,09. A média e o desvio-padrão de cada item que compõe os indicadores de avaliação do serviço e de satisfação com o AASI, estão descritos na Tabela 4.

$\mathrm{Na}$ análise bivariada, faixa etária, ocupação e nível de orientação estiveram associados à satisfação com o AASI. Quando analisadas em conjunto, por meio da regressão logística, apenas a variável "orientações quanto ao uso do AASI" esteve associada à satisfação, sendo que a insatisfação com o AASI 
Tabela 2. Acessibilidade ao Serviço de Atenção à Saúde Auditiva

\begin{tabular}{|c|c|c|}
\hline Variáveis & $\mathrm{n}$ & $\%$ \\
\hline \multicolumn{3}{|l|}{ Forma de acesso ao serviço } \\
\hline Encaminhado formalmente & 117 & 38,7 \\
\hline Recebeu orientação & 138 & 45,7 \\
\hline Procura espontânea & 47 & 15,6 \\
\hline \multicolumn{3}{|c|}{ Tempo de espera para atendimento } \\
\hline 0 a 3 meses & 206 & 68,2 \\
\hline 3 a 6 meses & 55 & 18,2 \\
\hline 6 a 12 meses & 26 & 8,6 \\
\hline$>12$ meses & 15 & 4,9 \\
\hline \multicolumn{3}{|c|}{ Teste com mais de um tipo/marca de AASI } \\
\hline Sim & 57 & 18,9 \\
\hline Não & 245 & 81,1 \\
\hline \multicolumn{3}{|l|}{ Tempo de espera pelo AASI } \\
\hline Menos de 1 mês & 93 & 30,8 \\
\hline 1 a 3 meses & 164 & 54,3 \\
\hline 3 a 6 meses & 35 & 11,6 \\
\hline$>6$ meses & 10 & 3,3 \\
\hline \multicolumn{3}{|c|}{ Retorno ao SASA para acompanhamento* } \\
\hline Sim & 98 & 46,4 \\
\hline Não & 113 & 53,6 \\
\hline \multicolumn{3}{|l|}{ Avaliação global pelo usuário } \\
\hline Ótimo & 192 & 63,6 \\
\hline Bom & 102 & 33,8 \\
\hline Regular & 5 & 1,7 \\
\hline Ruim & 3 & 1,0 \\
\hline
\end{tabular}

*Foram considerados nesta variável apenas os entrevistados com tempo igual ou superior a um ano de recebimento do aparelho, contado até a data da entrevista, período em que o retorno ao serviço é necessário - ou deveria ser compulsório
Tabela 3. Uso e manutenção do AASI de usuários do SUS

\begin{tabular}{|c|c|c|}
\hline Variáveis & $\mathrm{n}$ & $\%$ \\
\hline \multicolumn{3}{|l|}{ Uso do AASI durante a visita } \\
\hline Sim & 105 & 34,8 \\
\hline Não & 197 & 65,2 \\
\hline \multicolumn{3}{|l|}{ Uso prévio de outro AASI } \\
\hline Sim & 67 & 22,2 \\
\hline Não & 235 & 77,8 \\
\hline \multicolumn{3}{|l|}{ Orientações quanto ao uso do AASI } \\
\hline Sente-se suficientemente orientado & 223 & 73,8 \\
\hline Tem dúvidas em como usar o AASI & 79 & 26,2 \\
\hline \multicolumn{3}{|l|}{ Tempo de uso diário do AASI } \\
\hline O dia todo & 98 & 32,5 \\
\hline Mais da metade do dia & 33 & 10,9 \\
\hline Menos da metade do dia & 32 & 10,6 \\
\hline Não está usando & 139 & 46,0 \\
\hline \multicolumn{3}{|l|}{ Satisfação com AASI } \\
\hline Sim & 246 & 81,5 \\
\hline Não & 56 & 18,5 \\
\hline \multicolumn{3}{|l|}{ Reposição da pilha } \\
\hline Sem dificuldade & 225 & 74,5 \\
\hline Dificuldade financeira & 33 & 10,9 \\
\hline Dificuldade devido à distância & 29 & 9,6 \\
\hline Dificuldade financeira e distância & 8 & 2,6 \\
\hline Manuseio da pilha & 7 & 2,3 \\
\hline \multicolumn{3}{|l|}{ Percepção de melhora na vida após AASI } \\
\hline Sim & 239 & 79,1 \\
\hline Não sentiu melhora & 63 & 20,9 \\
\hline
\end{tabular}

Tabela 4. Média e desvio-padrão dos indicadores de satisfação dos usuários com o AASI e com o serviço $(n=302)$

\begin{tabular}{lccc}
\hline Critério & Pontuação máxima & Média & Desvio-padrão \\
\hline Avaliação do Serviço & 10 & 7,25 & 1,370 \\
Teste com mais de um tipo/marca de AASI & 1 & 0,19 & 0,392 \\
Tempo de espera pelo AASI & 3 & 2,81 & 1,65 \\
Retorno ao serviço após recebimento do AASI & 3 & 2,60 & 0,481 \\
Avaliação global pelo usuário & 3 & 5,09 & 3,579 \\
Satisfação com o aparelho & 10 & 2,09 & 2,152 \\
Uso atual do AASI & 5 & 3,00 & 1,774 \\
Melhora na vida após uso do AASI & 5 & & \\
\hline
\end{tabular}

foi maior entre aqueles que referiam dúvidas quanto ao uso do dispositivo. As associações entre o indicador de satisfação, as variáveis sociodemográficas e as variáveis relacionadas ao uso do AASI, estão apresentadas na Tabela 5.

A pesquisa de correlação entre o indicador de avaliação do serviço e o indicador de satisfação com o AASI foi significativa $(\mathrm{p}<0,001)$, porém fraca $(\mathrm{rho}=0,245)$.

\section{DISCUSSÃO}

O presente estudo possibilitou avaliar o Serviço de Atenção à Saúde Auditiva (SASA) e o resultado da adaptação de AASI, na perspectiva dos usuários. A média do indicador de satisfação dos usuários com o serviço foi classificada como "boa", enquanto que, com o aparelho de amplificação sonora, 
Tabela 5. Associação entre satisfação com o AASI e variáveis sociodemográficas

\begin{tabular}{|c|c|c|c|c|c|c|c|c|c|}
\hline \multirow{3}{*}{ Variáveis } & \multicolumn{5}{|c|}{ Indicador de satisfação com o AASI } & \multirow{3}{*}{ OR bruta } & \multirow{3}{*}{ IC (95\%) } & \multirow{3}{*}{ OR ajustada } & \multirow{3}{*}{ IC (95\%) } \\
\hline & \multicolumn{2}{|c|}{ Satisfeito } & \multicolumn{2}{|c|}{ Insatisfeito } & \multirow{2}{*}{ Valor de $p$} & & & & \\
\hline & $(n)$ & $\%$ & $(n)$ & $\%$ & & & & & \\
\hline \multicolumn{10}{|l|}{ Gênero } \\
\hline Feminino & 84 & 50 & 84 & 50 & & & & & \\
\hline Masculino & 59 & 44 & 75 & 56 & 0,302 & 1,27 & $0,81-2,00$ & & \\
\hline \multicolumn{10}{|l|}{ Faixa etária } \\
\hline$<60$ & 50 & 56,8 & 38 & 43,2 & & & & & \\
\hline$\geq 60$ & 93 & 43,5 & 121 & 56,5 & 0,035 & 1,71 & $1,04-2,82$ & 1,12 & $0,58-2,16$ \\
\hline \multicolumn{10}{|l|}{ Escolaridade } \\
\hline$\geq 8$ anos & 48 & 55,8 & 38 & 44,2 & & & & & \\
\hline$<8$ anos & 95 & 44 & 121 & 56 & 0,063 & 1,61 & $0,97-2,66$ & 1,07 & $0,59-1,95$ \\
\hline \multicolumn{10}{|l|}{ Ocupação } \\
\hline Trabalha & 39 & 59,1 & 27 & 40,9 & & & & & \\
\hline Não trabalha/aposentado & 104 & 44,1 & 132 & 55,9 & 0,031 & 1,83 & $1,05-3,19$ & 1,22 & $0,65-2,29$ \\
\hline \multicolumn{10}{|l|}{ Tipo AASI } \\
\hline Extraural & 107 & 45,3 & 129 & 54,7 & & & & & \\
\hline Intraural & 36 & 54,5 & 30 & 45,5 & 0,185 & 0,69 & $0,40-1,20$ & 1,07 & $0,52-2,20$ \\
\hline \multicolumn{10}{|l|}{ Categoria } \\
\hline C & 86 & 52,8 & 77 & 47,2 & & & & & \\
\hline$A / B$ & 37 & 42 & 51 & 58 & 0,105 & 1,54 & $0,91-2,60$ & 1,53 & $0,90-2,61$ \\
\hline \multicolumn{10}{|l|}{ Uso prévio de outro AASI } \\
\hline Não & 105 & 44,7 & 130 & 55,3 & & & & & \\
\hline Sim & 38 & 56,7 & 29 & 43,3 & 0,082 & 0,62 & $0,36-1,07$ & 0,63 & $0,32-1,21$ \\
\hline \multicolumn{10}{|l|}{ Orientações } \\
\hline Sente-se orientado & 120 & 53,8 & 103 & 46,2 & & & & & \\
\hline Tem dúvidas & 23 & 29,1 & 56 & 70,9 & $<0,001$ & 2,84 & $1,63-4,93$ & $2,73^{*}$ & $1,51-4,93$ \\
\hline \multicolumn{10}{|l|}{ Adaptação } \\
\hline Bilateral & 128 & 46,7 & 146 & 53,3 & & & & & \\
\hline Unilateral & 15 & 53,6 & 13 & 46,4 & 0,489 & 0,76 & $0,35-1,66$ & & \\
\hline \multicolumn{10}{|l|}{ Teste com marcas } \\
\hline Sim & 24 & 42,1 & 33 & 57,9 & & & & & \\
\hline Não & 119 & 48,6 & 126 & 51,4 & 0,379 & 0,77 & $0,43-1,38$ & & \\
\hline
\end{tabular}

*Valor significativo $(p<0,05)$ - Regressão logística binária

Legenda: $\mathrm{OR}=$ Odds Ratio; IC = Intervalo de Confiança

foi classificada como "regular". Salientamos esse tópico, no sentido de demonstrar que a literatura não registra estudos que avaliem simultaneamente a satisfação com o serviço e com o aparelho, sendo escassas as avaliações de serviços ${ }^{(13)}$. Pesquisas que utilizaram outros instrumentos para avaliar a satisfação dos usuários com seu aparelho auditivo ${ }^{(9,15-17)}$, revelaram níveis mais elevados do que os do presente estudo. Dentro da visão de controle de qualidade no atendimento dos profissionais da saúde, aponta-se a medida da satisfação do usuário como um dos fatores que devem ser analisados. É importante avaliar os efeitos proporcionados pelo uso do AASI e dos serviços, com o objetivo de melhorar a prática assistencial em Audiologia ${ }^{(18)}$.
Em um estudo brasileiro ${ }^{(9)}$ e outro americano ${ }^{(17)}$, que utilizaram o IOI-HA, a maioria dos usuários fez uso efetivo do AASI e declarou estar satisfeita com o aparelho. Em outro estudo ${ }^{(15)}$, que utilizou o questionário Satisfaction with Amplification in Daily Life (SADL), os usuários registraram alta pontuação em todas as subescalas do instrumento. No entanto, tais pesquisas apresentam amostra muito restrita, quando comparadas ao presente estudo.

Outro aspecto que poderia justificar a baixa pontuação do indicador de satisfação com o aparelho auditivo, foi a elevada proporção dos indivíduos que não estava fazendo uso do dispositivo. Em tais situações, o escore relativo à satisfação com o AASI foi igual a zero, o que impactou no resultado final. Uma 
alta proporção de pessoas que não fazem uso de AASI já foi destacada em outro estudo ${ }^{(19)}$.

Salienta-se, todavia, a particularidade do instrumento, que, além de ter aferido o escore de satisfação, aferiu a percepção subjetiva do usuário em relação à satisfação com o AASI. Essa característica revelou uma situação paradoxal, pois o número de pessoas que se disseram satisfeitas com o dispositivo foi muito alto, indicando que a satisfação nem sempre está associada ao benefício proporcionado pelo AASI, ou à sua efetiva utilização ${ }^{(20)}$.

Quando questionado o motivo da satisfação, dentre aqueles que não estavam usando o dispositivo, a maioria respondeu que o aparelho auxiliou bastante, que passaram a escutar melhor com ele. Entretanto, esse benefício proporcionado pelo aparelho referia-se à época em que usavam, ou seja, os indivíduos se mostraram satisfeitos por um benefício que tiveram no passado, mesmo sabendo que o aparelho não representava alguma ajuda quando foram entrevistados. Pesquisadores ${ }^{(21)}$ comentam que o medo de perder o acesso e a afinidade com os profissionais do serviço podem levar os usuários a omitirem opiniões negativas, refletindo um viés na satisfação.

Uma boa parcela dos entrevistados informou, ainda, que estava satisfeita por ter recebido o aparelho gratuitamente. Alguns, inclusive, associaram esse fato a um acontecimento de sorte. Esse sentimento de gratidão é observável em usuários do Sistema Único de Saúde, que não se veem como cidadãos com direitos perante o serviço público, o que dificulta a avaliação da qualidade do dispositivo, sob a perspectiva do usuário ${ }^{(12)}$. Esse aspecto pode ser mais percebido nas regiões mais carentes, como o local do estudo, por exemplo, que concentra várias cidades com baixo Índice de Desenvolvimento Humano (IDH).

Os efeitos proporcionados pelos aparelhos de amplificação sonora podem variar entre os indivíduos e a literatura registra diferenças que variam de acordo com a região geográfica. Em Santa Maria - RS, o uso do AASI foi efetivo e o benefício bastante satisfatório entre os usuários ${ }^{(9)}$, assim como nos municípios de Recife $(\mathrm{PE})^{(22)}$ e Lauro de Freitas $(\mathrm{BA})^{(23)}$, em que o nível de satisfação com o aparelho foi alto. Em Bauru (SP), dois estudos registraram que os pesquisados também estavam satisfeitos com seus aparelhos auditivos ${ }^{(24,25)}$. Entretanto, um desses estudos observou que uma parcela significativa da amostra não havia retornado para acompanhamento e alguns não estavam fazendo uso efetivo do AASI ${ }^{(24)}$. Em João Pessoa $(\mathrm{PB})$, os indivíduos assistidos pelo SUS, em geral, não obtiveram resultados satisfatórios com a amplificação. Dificuldades com relação ao uso e manuseio foram identificadas, assim como tempo reduzido de uso diário dos aparelhos, dificultando a obtenção do benefício ${ }^{(14)}$. É importante destacar que os instrumentos utilizados para avaliar a satisfação são diferentes em alguns estudos, além de contemplarem uma amostra pequena, em sua maioria.

Queixas sobre dificuldades de adaptação ao AASI, similares às relatadas pela maioria das pessoas do presente estudo que não estavam fazendo uso do aparelho, como incômodos com a amplificação causados por ruídos, cefaleias, prurido, entre outros, também foram mencionadas em outro estudo ${ }^{(26)}$ e demandam adequado acompanhamento do serviço de saúde para serem investigadas e sanadas.

O impacto que o uso do aparelho auditivo proporciona na vida das pessoas é diversificado, dependendo de suas condições físicas, emocionais, sociais e culturais ${ }^{(20)}$. Enquanto para alguns o aparelho tem representado expressiva melhora na qualidade de vida, para outros, o impacto não tem sido observado de forma significativa.

As referências a defeitos no dispositivo, ou dificuldades para adquirir a pilha, por impossibilidade de acesso, ou por questões financeiras, apontadas por alguns pacientes do presente estudo como motivos para não fazer uso do AASI, podem ser baseadas no fato de que, apesar de terem a reabilitação auditiva garantida pelo SUS, existem custos monetários envolvidos na manutenção do aparelho, de responsabilidade do paciente, o que justificaria a demora pela procura em solucionar os problemas encontrados ${ }^{(26)}$. Mesmo entre os que estavam fazendo uso do AASI, alguns usuários relataram não fazê-lo em tempo integral para "economizar" as pilhas. A dificuldade para repor a pilha devido ao acesso foi observada, principalmente, em municípios menores.

Embora a maioria dos entrevistados tenha relatado que se sente suficientemente orientada a usar o aparelho auditivo, a associação entre satisfação e orientações sobre como usar o AASI, identificada por meio da regressão logística, revela que as dificuldades em manusear $\mathrm{o}$ aparelho interferem diretamente no uso efetivo do AASI ${ }^{(2,14)}$. Tal resultado reflete a importância da participação ativa da família no programa de reabilitação ${ }^{(27)}$ e destaca a relevância do acesso à informação, que poderia ser melhor obtido a partir do acompanhamento sistemático do paciente.

O indicador de satisfação com o serviço, em que os escores obtidos nas entrevistas com os usuários o conceituaram como "bom", sendo que mais da metade dos usuários referiu o atendimento global como "ótimo", elogiando o tratamento recebido pelos profissionais, concorda com os resultados do projeto multicêntrico desenvolvido em Bauru, com o intuito de elaborar um instrumento que avaliasse a qualidade do serviço de saúde auditiva. Nesse instrumento, os usuários do SUS também afirmaram estar satisfeitos com o serviço, e os melhores escores do questionário foram referentes à competência profissional, avaliação da audição e atendimento personalizado ${ }^{(13)}$. Pacientes que estão satisfeitos com seus aparelhos auditivos também se sentem gratos aos profissionais que os ofertaram ${ }^{(17)}$. A correlação observada entre o indicador de avaliação do serviço e o indicador de satisfação com o AASI apresentou-se positiva e significativa, porém, justificando apenas uma parcela dos resultados, já que a correlação foi fraca.

A média do indicador de avaliação do serviço foi classificada como "boa" porque a grande maioria dos usuários não fez testes 
com outros tipos/marcas de aparelho e muitos não retornaram ao serviço após recebimento do AASI. A portaria SAS/MS n 587 de 2004, do Ministério da Saúde, que regulamenta os serviços de saúde auditiva recomenda testar três tipos e/ou marcas de aparelho no momento da seleção. Embora a falta de testes com diferentes tipos e marcas possa contribuir com as dificuldades de adaptação dos usuários ${ }^{(9)}$, neste estudo, esta variável não se mostrou associada.

O grande número de pessoas que não retornou para acompanhamento no Serviço é um fato que merece atenção. Alguns indivíduos relataram desconhecer que deveriam retornar após um ano, dizendo também que não foram chamados. Esse acompanhamento anual, no caso de adultos e idosos, também é estabelecido por portaria ministerial. Ao término da terapia fonoaudiológica, o paciente recebe uma guia com informações sobre o acompanhamento, que pode ser agendado pelo fonoaudiólogo, descentralizado/referência técnica do município, ou pelo próprio serviço. Pesquisadores realizaram levantamento do quantitativo dos procedimentos relacionados à adaptação de AASI no Brasil, e constataram que o acompanhamento fonoaudiológico ainda é pouco realizado. Assim, os serviços precisam se organizar para investigar os motivos da não realização deste procedimento em pacientes adaptados com $\mathrm{AASI}^{(6)}$.

O AASI é o início do processo de reabilitação das pessoas com perda auditiva, mas a terapia após a adaptação é fundamental para reduzir as barreiras de comunicação. Usuários de AASI submetidos à reabilitação com treinamento das habilidades auditivas tiveram benefício maior, quando comparados aos que não foram submetidos aos mesmos procedimentos ${ }^{(28)}$, aumentando o uso efetivo do AASI, utilizando mais estratégias de comunicação no dia a dia e melhorando sua qualidade de vida $^{(29)}$. Dessa forma, muitos dos "incômodos" relatados pelos entrevistados poderiam ser solucionados nesses encontros. A orientação ao paciente, antes e após a adaptação, também é essencial para diminuir sua desvantagem auditiva e sua ansiedade $^{(30)}$, pois expectativas excessivas com relação ao uso do AASI podem levar à frustração e abandono do dispositivo.

Estratégias que minimizem a falta de adesão ao tratamento precisam ser desenvolvidas, para que os recursos gastos com os indivíduos deficientes auditivos não culminem em desperdício, conforme já destacado por outro estudo ${ }^{(24)}$. A articulação entre a Atenção Primária à Saúde e o Serviço de Média e Alta Complexidade pode ser uma dessas estratégias, pois garantiria atenção integral ao paciente. A capacitação de Agentes Comunitários de Saúde pode ser uma solução viável para educação continuada aos usuários de AASI. Novos estudos devem ser conduzidos para avaliar essa possibilidade.

São necessários, também, novos estudos para ampliar a acurácia no processo de avaliação da satisfação dos usuários, já que os estudos existentes registram importantes diferenças entre si. O questionário utilizado neste estudo foi considerado de fácil aplicação e compreensão, sendo útil para o controle de qualidade do serviço. Instrumentos que avaliem a qualidade do serviço em outros seguimentos, como a estrutura, e com diferentes atores envolvidos no processo, como profissionais e gestores, devem ser utilizados, com o objetivo de garantir uma atenção eficiente. A avaliação dos serviços de saúde auditiva faz-se necessária como forma de acrescentar melhorias à Política de Atenção à Saúde Auditiva ${ }^{(12)}$.

Os resultados deste estudo devem ser considerados à luz de algumas limitações. Não foi possível, nesta pesquisa, contar com todos os dados necessários para uma análise profunda, com relação ao tipo e intensidade da perda auditiva. Significativa parcela dos pacientes não dispunha de cópias dos seus exames, o que está em desacordo com a legislação vigente. $\mathrm{O}$ fato de o estudo ter utilizado um instrumento ainda pouco difundido também limita as análises comparativas com outros estudos. Uma alternativa para contornar as limitações encontradas seria a realização de um estudo longitudinal, em pesquisas posteriores. Deve-se destacar, todavia, que o presente estudo contemplou uma grande amostra, representativa de extensa área geográfica, com análise de dados coletados diretamente com os pacientes.

\section{CONCLUSÃO}

Os resultados obtidos através do indicador de satisfação com o AASI apresentaram nível abaixo do que registram outros estudos, decorrente do alto número de indivíduos que não estava fazendo uso do aparelho. A insatisfação foi maior entre aqueles que tinham dúvidas com relação ao uso e manuseio do aparelho. Os desconfortos percebidos foram causas dos problemas na adaptação e precisam ser mais bem investigadas e sanadas.

Ações previstas pela legislação, como testes com diferentes tipos e/ou marcas de aparelho e acompanhamento anual, não foram registradas nas entrevistas com a maioria dos usuários assistidos no serviço. Contudo, estão satisfeitos com os profissionais e serviço ofertado, podendo ser resultado do sentimento de gratidão, comum entre usuários do SUS.

\section{REFERÊNCIAS}

1. Instituto Brasileiro de Geografia e Estatística. Censo Demográfico 2010: resultados gerais da amostra. Rio de Janeiro: IBGE, 2010.

2. Hampson, R. Hearing aids. Eur Geriatr Med. 2012;3(3):198-200.

3. Peelle JE, Troiani V, Grossman M, Wingfield A. Hearing loss in older adults affects neural systems supporting speech comprehension. J Neurosci. 2011;31(35):12638-43.

4. Espmark AK, Rosenhall U, Erlandsson S, Steen B. The two faces of presbyacusis: hearing impairment and psychosocial consequences. Int J Audiol. 2002;41(2):125-35.

5. Pacala JT, Yueh B. Hearing deficits in the older patient: "I didn't notice anything". JAMA. 2012;307(11):1185-94.

6. Bevilacqua MC, Morettin M, Melo TM, Amantini RCB, Martinez MANS. Contribuições para análise da política de saúde auditiva no Brasil. Rev Soc Bras Fonoaudiol. 2011;16(3):252-9. 
7. Rossi-Barbosa LAR, Vieira SMFV, Manzoni CRT, Caldeira AP, Honorato-Marques R, Ribeiro JA, et al. Processo de construção de indicadores em saúde auditiva. Rev CEFAC. 2011;13(1):102-11.

8. Cook JA, Hawkins DB. Outcome measurement for patients receiving hearing aid services. Laryngoscope. 2007;117(4):610-13.

9. Lopes AS, Costa MJ, Aurélio NHS, Santos SN, Vaucher AV. A satisfação e o desempenho de usuários de próteses auditivas atendidos em um programa de atenção à saúde auditiva. Rev CEFAC. 2011;13(4):698-709.

10. Cox R, Hyde M, Gatehouse S, Noble W, Dillon H, Bentler R, et al. Optimal outcome measure, research priorities and international cooperation. Ear Hear. 2000;21(4 Suppl):S106-S115.

11. Gasparin M, Menegotto IH, Cunha CS da. Propriedades psicométricas do questionário internacional: aparelho de amplificação sonora individual. Braz J Otorhinolaryngol. 2010;76(1):85-90.

12. Bevilacqua MC, Melo TM, Morettin M, Lopes AC. A avaliação de serviços em Audiologia: concepções e perspectivas. Rev Soc Bras Fonoaudiol. 2009;14(3):421-6.

13. Armigliato ME, Prado DGA, Melo TM, Martinez MANS, Lopes AC, Amantini RCB, et al. Avaliação de serviços de saúde auditiva sob a perspectiva do usuário: proposta de instrumento. Rev Soc Bras Fonoaudiol. 2010;15(1):32-9.

14. Buriti AKL, Oliveira SHS. Adaptação à prótese auditiva em usuários assistidos pelo Sistema Único de Saúde. Rev Soc Bras Fonoaudiol. 2012;17(1):41-6.

15. Danieli F, Castiquini EAT, Zambonatto TCF, Bevilacqua MC. Avaliação do nível de satisfação de usuários de aparelhos de amplificação sonora individuais dispensados pelo Sistema Único de Saúde. Rev Soc Bras Fonoaudiol. 2011;16(2):152-9.

16. Heuermann H, Kinkel M, Tchorz J. Comparison of psychometric of the International Outcome Inventory for Hearing Aids (IOI-HA) in various studies. Int J Audiol. 2005;44(2):102-9.

17. Williams VA, Johnson CE, Danhauer JL. Hearing aid outcomes: effects of gender and experience on patients' use and satisfaction. J Am Acad Audiol. 2009;20(7):422-32.

18. Hickson L, Clutterbuck S, Khan A. Factors associated with hearing aid fitting outcomes on the IOI-HA. Int J Audiol. 2010;49(8):586-95.

19. Hartley D, Rochtchina E, Newall P, Golding M, Mitchell P. Use of hearing aids and assistive listening devices in an older Australian population. J Am Acad Audiol. 2010;21:642-53.
20. Assayag FH, Russo ICP. Avaliação subjetiva do benefício e dos efeitos proporcionados pelo uso de amplificação sonora em indivíduos idosos. Distúrb Comun. 2006;18(3):383-90.

21. Espiridião M, Trad LAB. Avaliação de satisfação dos usuários. Ciênc Saúde Colet. 2005;10(Suppl):303-12.

22. Teixeira CF, Augusto LGS, Caldas Neto CC. Prótese auditiva: satisfação do usuário com sua prótese e com seu meio ambiente. Rev CEFAC. 2008;10(2):245-53.

23. Farias RB, Russo ICP. Saúde auditiva: estudo do grau de satisfação de usuários de aparelho de amplificação sonora individual. Rev Soc Bras Fonoaudiol. 2010;15(1):26-31.

24. Morettin M. Avaliação dos benefícios e satisfação dos usuários de Aparelho de Amplificação Sonora Individual nos serviços de Audiologia do SUS [dissertação]. São Paulo: Universidade de São Paulo; 2008.

25. Picolini MM, Blasca WQ, Campos K, Mondelli MFCG. Adaptação aberta: avaliação da satisfação dos usuários de um centro de alta complexidade. Rev CEFAC. 2011;13(4):676-84.

26. Freitas CD, Costa MJ. Processo de adaptação de próteses auditivas em usuários atendidos em uma instituição pública federal - parte I: resultados e implicações com o uso da amplificação. Rev Bras Otorrinolaringol. 2007;73(6):744-51.

27. Miranda EC, Calais LL, Vieira EP, Carvalho LMA, Borges ACLC, Iório MCM. Dificuldades e benefícios com o uso de prótese auditiva: percepção do idoso e sua família. Rev Soc Bras Fonoaudiol. 2008;13(2):166-72.

28. Megale RL, Iório MCM, Schochat E. Treinamento auditivo: avaliação do benefício em idosos usuários de próteses auditivas. Pró-Fono. 2010;22(2):101-6.

29. Araújo TM, Morettin M, Torres KCC, Brasil HK, Silva TA, Laperuta EB et al. Relação entre o processo de reabilitação auditiva e a auto-percepção da qualidade de vida em usuários de Aparelho de Ampliação Sonora Individual: revisão sistemática. Distúrb Comun. 2010;22(1):25-36.

30. Lundberg M, Andersson G, Lunner T. A randomized, controlled trial of the short-term effects of complementing an educational program for hearing aid users with telephone consultations. J Am Acad Audiol. 2011;22(10):654-62. 